\title{
POSITIVE FUNCTIONALS INDUCED BY MINIMIZERS OF CAUSAL VARIATIONAL PRINCIPLES
}

\author{
FELIX FINSTER
}

AUGUST 2017

Dedicated to the memory of Eberhard Zeidler

\begin{abstract}
Considering second variations about a given minimizer of a causal variational principle, we derive positive functionals in space-time. It is shown that the strict positivity of these functionals ensures that the minimizer is nonlinearly stable within the class of compactly supported variations with local fragmentation. As applications, we endow the space of jets in space-time with Hilbert space structures and derive a positive surface layer integral on solutions of the linearized field equations.
\end{abstract}

\section{Contents}

1. Introduction

2. Preliminaries: Minimizers of Causal Variational Principles

3. Positivity of the Hessian of $\ell$

4. Positivity of Second Variations Generated by Jets

5. Stability under Variations with Local Fragmentation

6. Application: Hilbert Spaces of Jets

7. Application: A Positive Surface Layer Integral References

\section{INTRODUCTION}

Given a minimizer of a variational principle, second variations are always nonnegative. This basic observation goes back to Legendre and Jacobi, who used it to analyze the question whether classical trajectories minimize the action and geodesics minimize arc length [11. In classical field theory (like electrodynamics or general relativity), second variations are less useful because in these theories the action is unbounded from below, so that instead of minimizing one merely seeks for critical points of the action. However, in the recent theory of causal fermion systems (see the textbook [3] or the physical introduction [7]), the action is indeed bounded from below, and physical space-time should be described by a minimizer. Therefore, second variations should give rise to positive functionals in space-time. In special situations and for specific variations (so-called scalar variations; see Remark 4.2 below), such functionals have already been obtained in [10, Lemma 3.5] and [1, Sections 4.4 and 4.5]. Here we give a more general construction which gives rise to two positive functionals. We show that these functionals describe the local behavior of the action completely, in the sense that if the functionals are strictly positive, then the minimizer is nonlinearly stable within a well-defined general class of variations. Moreover, it is shown that our 
positive functionals can be used to endow the space of jets in space-time with Hilbert space structures. Finally, our functionals give rise to a positive surface layer integral on solutions of the linearized field equations.

In general terms, in a causal variational principle one minimizes an action $\mathcal{S}$ of the form

$$
\mathcal{S}(\rho)=\int_{\mathcal{F}} d \rho(x) \int_{\mathcal{F}} d \rho(y) \mathcal{L}(x, y)
$$

under variations of the measure $\rho$, keeping the total volume $\rho(\mathcal{F})$ fixed (volume constraint). In order to keep the presentation self-contained and reasonably simple, we here restrict attention to the smooth setting as considered in [9. Section 3] (but all our arguments and results can be generalized in a straightforward manner to the lower-semicontinuous setting by distinguishing vector fields pointing in directions in which directional derivatives exist; for details see [9, Section 4]). Thus we let $\mathcal{F}$ be a (possibly non-compact) smooth manifold of dimension $m \in \mathbb{N}$. Moreover, the $L a$ grangian $\mathcal{L} \in C^{\infty}\left(\mathcal{F} \times \mathcal{F}, \mathbb{R}_{0}^{+}\right)$is given as a smooth non-negative function which is symmetric (i.e. $\mathcal{L}(x, y)=\mathcal{L}(y, x)$ for all $x, y \in \mathcal{F})$. Let $\rho$ be a minimizing measure (for details see Section 2 below). We refer to the support of the measure as

$$
\text { space-time } \quad M:=\operatorname{supp} \rho .
$$

The notion causal in "causal variational principles" refers to the fact that the Lagrangian induces on $M$ a causal structure. Namely, two space-time points $x, y \in M$ are said to be timelike and space-like separated if $\mathcal{L}(x, y)>0$ and $\mathcal{L}(x, y)=0$, respectively. For more details on this notion of causality, its connection to the causal structure in Minkowski space and to general relativity we refer to [3, Chapter 1], [5] and [3, Sections 4.9 and 5.4].

First variations can be computed with the help of the formula

$$
\delta S=2 \int_{\mathcal{F}} \ell(x) d \delta \rho(x) \quad \text { with } \quad \ell(x):=\int_{\mathcal{F}} \mathcal{L}(x, y) d \rho(y)-\frac{\nu}{2},
$$

where $\nu$ is an arbitrary real parameter. The condition that this variation be nonnegative for any variation $\delta \rho$ which respects the volume constraint implies that the function $\ell$ is minimal and constant on $M$ (for details see again Section 2 below). We always choose the real parameter $\nu$ such that this constant is zero, i.e.

$$
\left.\ell\right|_{M} \equiv \inf _{\mathcal{F}} \ell=0
$$

This means in words that when minimizing the action, the measure $\rho$ is driven towards the minima of the function $\ell$, which in turn are arranged to all have the same value of $\ell$. As a result, space-time $M$ will typically be a discrete or low-dimensional subset of $\mathcal{F}$ (this picture has been confirmed by numerical and analytic results in [10]).

For the variations we consider families of measures $\left(\tilde{\rho}_{\tau}\right)_{\tau \in\left[0, \tau_{\max }\right)}$ with $\tau_{\max }>0$ such that $\tilde{\rho}_{0}$ coincides with our minimizer $\rho$. We now explain how to choose the class of variations. First of all, the family should satisfy the volume constraint (as will be made precise again in Section 2 below). Moreover, if the first variation is strictly positive, then the second variation need not be considered. Thus in view of (1.2) and (1.3), it suffices to consider the situation that the support of the varied measures lies in the set where $\ell$ is small, i.e. given any $\varepsilon>0$ we may assume that

$$
\operatorname{supp} \tilde{\rho}_{\tau} \subset \ell^{-1}([0, \varepsilon)) \quad \text { for all } \tau \in\left[0, \tau_{\max }\right) \text {. }
$$



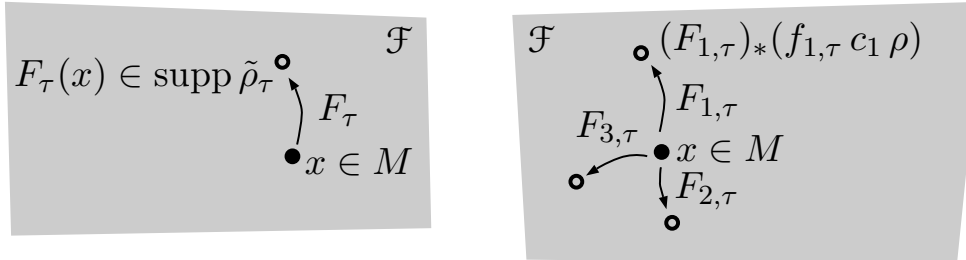

FiguRE 1. Varying the measure with fragmentation.

There is the subtle issue that the set $\ell^{-1}([0, \varepsilon))$ may contain points which are not in a small neighborhood of $M$, no matter how small we choose $\varepsilon$. For example, there may be points $y \in \mathcal{F} \backslash M$ with $\ell(y)=0$. This situation has been analyzed in [1, Section 3.5], and we will discuss it in Remark 4.3 below. Moreover, there might be sequences $\left(y_{n}\right)_{n \in \mathbb{N}}$ with $\ell\left(y_{n}\right) \searrow 0$ which have no accumulation points (note that $\mathcal{F}$ may be non-compact). This would mean intuitively that " $\ell$ has a minimum at infinity," implying that (1.4) would not give us control of the support of $\tilde{\rho}_{\tau}$ for small $\tau$. This case, which seems difficult and somewhat artificial, will not be covered by our analysis. Instead, we shall only consider variations supported in a small neighborhood of $M$, i.e. for any open neighborhood $U$ of $M$ we assume that by decreasing $\tau_{\max }$ we can arrange that

$$
\operatorname{supp} \tilde{\rho}_{\tau} \subset U \quad \text { for all } \tau \in\left[0, \tau_{\max }\right) \text {. }
$$

A method for varying measures which has been proven fruitful in [9] is to multiply $\rho$ by a positive smooth weight function $f_{\tau}$ and then to take the push-forward under a smooth mapping $F_{\tau}: M \rightarrow \mathcal{F}$. This leads to the ansatz (for details see Section 4 below)

$$
\tilde{\rho}_{\tau}=\left(F_{\tau}\right)_{*}\left(f_{\tau} \rho\right) .
$$

Since multiplying by a positive function leaves the support unchanged, the support of the measure is transformed only by $F_{\tau}$; more precisely,

$$
\operatorname{supp} \tilde{\rho}_{\tau}=\overline{F_{\tau}(\operatorname{supp} \rho)} \text {. }
$$

In particular, for this family of measures, the support changes smoothly in $\tau$. For example, if $M$ is discrete, then the measures $\rho_{\tau}$ will necessarily also be discrete (see the left of Figure 1 for the example of a Dirac measure). However, this is a much stronger condition than (1.5), according to which a measure supported at a point $x \in M$ could "disintegrate" in the variation and become for example a weighted sum of Dirac measures supported in a small neighborhood of $x$ (see the right of Figure 11). In order to take such effects into account, using the notion first introduced in [6, Section 5], we want to allow for the possibility of a fragmentation of the measure $\rho$. In mathematical terms, fragmentation is described as follows: Given a parameter $L \in \mathbb{N}$, we choose weight functions $c_{\mathfrak{a}}: M \rightarrow \mathbb{R}^{+}$for $a=1, \ldots, L$ which add up to one (for details see Section 5 )

$$
\sum_{\mathfrak{a}=1}^{L} c_{\mathfrak{a}}(x)=1 \quad \text { for all } x \in M
$$


Then we vary each of the measures $c_{\mathfrak{a}} \rho$ similar to (1.6) by functions $\left(f_{\mathfrak{a}, \tau}, F_{\mathfrak{a}, \tau}\right)$ (see again the right of Figure 1),

$$
\tilde{\rho}_{\tau}=\sum_{\mathfrak{a}=1}^{L}\left(F_{\mathfrak{a}, \tau}\right)_{*}\left(f_{\mathfrak{a}, \tau} c_{\mathfrak{a}} \rho\right) .
$$

Since $L$ can be chosen arbitrarily large, the resulting variations with fragmentation can be used to approximate all smooth variations which satisfy (1.5).

For technical simplicity, we restrict attention to variations which are compactly supported (i.e. trivial outside a compact set; for details see Section 4). Then the variation of the measure (1.6) is described infinitesimally by a pair of a real-valued function and a vector field on $M$ (for details see Section 3), both with compact support,

$$
(a, u):=\left.\frac{d}{d \tau}\left(f_{\tau}, F_{\tau}\right)\right|_{\tau=0} \in \mathfrak{J}_{0}^{\infty}:=C_{0}^{\infty}(M, \mathbb{R}) \oplus C_{0}^{\infty}(M, T \mathcal{F}) .
$$

The pair $\mathfrak{u}:=(a, u)$ is referred to as a jet. We denote the linear combination of multiplication and directional derivative by $\nabla$, i.e.

$$
\nabla_{\mathfrak{u}} g(x):=a(x) g(x)+\left(D_{u} g\right)(x)
$$

(where $g$ is a smooth function on $\mathcal{F}$ ). Then the EL equations (1.3) evaluated in a linear Taylor approximation on $M$ give rise to the so-called weak EL equations

$$
\left.\nabla_{\mathfrak{u}} \ell\right|_{M}=0 \quad \text { for all } \mathfrak{u} \in \mathfrak{J}_{0}^{\infty} .
$$

We now state our main result:

Theorem 1.1. Let $\rho$ be a minimizer of a causal variational principle in the noncompact smooth setting (for details see Section Q 2). Then the following two quadratic functionals on $\mathfrak{J}_{0}^{\infty}$ are positive:

$$
\begin{gathered}
\left.\int_{M} \nabla^{2} \ell\right|_{x}(\mathfrak{u}, \mathfrak{u}) d \rho(x) \geq 0 \\
\int_{M} d \rho(x) \int_{M} d \rho(y) \nabla_{1, \mathfrak{u}} \nabla_{2, \mathfrak{u}} \mathcal{L}(x, y)+\left.\int_{M} \nabla^{2} \ell\right|_{x}(\mathfrak{u}, \mathfrak{u}) d \rho(x) \geq 0 .
\end{gathered}
$$

Conversely, assume that $\rho$ is a Radon measure which satisfies (1.9) as well as the inequalities (1.10) and (1.11). If the inequality (1.11) is strict for every non-zero $\mathfrak{u} \in \mathfrak{J}_{0}^{\infty}$, then $\rho$ is an isolated local minimum within the class of compactly supported variations with local fragmentation (1.7).

The paper is organized as follows. In Section 2 we recall the necessary preliminaries on causal variational principles in the smooth setting. In Section 3, a positive functional is derived from the Hessian of $\ell$. Section 4 is devoted to another positive functional obtained by second variations generated by jets. In Section 5 the stability analysis is extended such as to allow for a fragmentation of the measure $\rho$. This will conclude the proof of Theorem 1.1. The last two sections are devoted to the applications: In Section [6 it is shown that the positive functionals endow the jet spaces with Hilbert space structures. Finally, in Section 7 a positive surface layer integral is derived.

I want to close with a personal remark. This paper is dedicated to the memory of Eberhard Zeidler, whom I admire both for his astounding knowledge and his deeply honest and amiable personality. I am very grateful and thankful for his guidance and advice. When I was post-doc at the Max Planck Institute for Mathematics in the 
Sciences in Leipzig from 1998 to 2002, we had long conversations in which he helped me to find the right direction of my research. In particular, I vividly remember that he encouraged me in my study of the interacting Dirac sea by telling me not to aim for short-term success or recognition by a scientific community. He told me that in the long run, thinking thoroughly on a problem would pay off. Taking this advice seriously led me to the physical theory of causal fermion systems and to the mathematical setting of causal variational principles, a certain aspect of which is presented here.

\section{Preliminaries: Minimizers of Causal Variational Principles}

Let $\mathcal{F}$ be a (possibly non-compact) smooth manifold of dimension $m \in \mathbb{N}$. Let $\mathcal{L} \in$ $C^{\infty}\left(\mathcal{F} \times \mathcal{F}, \mathbb{R}_{0}^{+}\right)$be a non-negative smooth function which is symmetric, i.e.

$$
\mathcal{L}(x, y)=\mathcal{L}(y, x) \quad \text { for all } x, y \in \mathcal{F} .
$$

We let $\rho$ be a Radon measure on $\mathcal{F}$ (i.e. a regular Borel measure with $\rho(K)<\infty$ for any compact $K \subset \mathcal{F}$, where by a measure we always mean a positive measure; for preliminaries see for example [12] or [2]). Moreover, we assume that $\rho$ satisfies the following technical assumption:

(a) For all $x \in \mathcal{F}$, the functions $\mathcal{L}(x,$.$) and \partial_{x^{j}} \mathcal{L}(x,$.$) are \rho$-integrable, giving smooth and bounded functions on $\mathcal{F}$. Moreover, partial derivatives and $y$-integration may be interchanged, i.e.

$$
\frac{\partial}{\partial x^{j}} \int_{M} \mathcal{L}(x, y) d \rho(y)=\int_{M} \frac{\partial \mathcal{L}(x, y)}{\partial x^{j}} d \rho(y) .
$$

If the total volume $\rho(\mathcal{F})$ is finite, the causal variational principle is to minimize the action (1.1) under variations of the measure $\rho$ (which do not need to satisfy (a)), keeping the total volume $\rho(\mathcal{F})$ fixed (volume constraint). If $\rho(\mathcal{F})$ is infinite, however, it is not obvious how to implement the volume constraint, making it necessary to proceed as follows: Let $\tilde{\rho}$ be another Borel measure on $\mathcal{F}$ (which again does not need not satisfy (a)) which has the properties

$$
|\tilde{\rho}-\rho|(\mathcal{F})<\infty \quad \text { and } \quad(\tilde{\rho}-\rho)(\mathcal{F})=0
$$

(where |.| denotes the total variation of a measure; see [12, §28] or [13, Section 6.1]). Then the difference of the actions as given by

$$
\begin{aligned}
& (\mathcal{S}(\tilde{\rho})-\mathcal{S}(\rho))=\int_{\mathcal{F}} d(\tilde{\rho}-\rho)(x) \int_{\mathcal{F}} d \rho(y) \mathcal{L}(x, y) \\
& \quad+\int_{\mathcal{F}} d \rho(x) \int_{\mathcal{F}} d(\tilde{\rho}-\rho)(y) \mathcal{L}(x, y)+\int_{\mathcal{F}} d(\tilde{\rho}-\rho)(x) \int_{\mathcal{F}} d(\tilde{\rho}-\rho)(y) \mathcal{L}(x, y)
\end{aligned}
$$

is well-defined (for details see [9, Lemma 2.1]). The measure $\rho$ is said to be a minimizer of the causal action if the difference (2.3) is non-negative for all $\tilde{\rho}$ satisfying (2.2),

$$
(\mathcal{S}(\tilde{\rho})-\mathcal{S}(\rho)) \geq 0 .
$$

We now state the Euler-Lagrange (EL) equations as derived in [9, Lemma 2.3] (by adapting [10, Lemma 3.4] to the non-compact setting). 
Lemma 2.1. (The Euler-Lagrange equations) Let $\rho$ be a minimizer of the causal action. Then for a suitable value of the real parameter $\nu$, the smooth function $\ell$ defined by

$$
\ell(x)=\int_{\mathcal{F}} \mathcal{L}(x, y) d \rho(y)-\frac{\nu}{2}: \mathcal{F} \rightarrow \mathbb{R}
$$

satisfies the equation

$$
\left.\ell\right|_{\operatorname{supp} \rho} \equiv \inf _{\mathcal{F}} \ell=0 .
$$

We remark that $\nu$ can be understood as the Lagrange multiplier describing the volume constraint; see [3, §1.4.1].

\section{Positivity of the Hessian of $\ell$}

Let $\rho$ be a minimizer of the causal action. According to the EL equations (2.5), the function $\ell$ is minimal on $M$. This clearly implies that its Hessian (as computed in any chart) is positive semi-definite, i.e.

$$
D^{2} \ell(x) \geq 0 \quad \text { for all } x \in M:=\operatorname{supp} \rho .
$$

This is the first non-negative quantity obtained from the fact that $\rho$ is a minimizer.

In order to clarify the connection to other non-negative functionals below, it is preferable to rewrite (3.1) in the jet formalism, which we now recall in the smooth setting. We denote the smooth vector fields on $\mathcal{F}$ restricted to $M$ by $C^{\infty}(M, T \mathcal{F})$ (where smoothness is defined by the condition that every $u \in C^{\infty}(M, T \mathcal{F})$ can be extended to a smooth vector field on $\mathcal{F}$ ). We introduce the smooth jet space $\mathfrak{J}^{\infty}$ by

$$
\mathfrak{J}^{\infty}=C^{\infty}(M, \mathbb{R}) \oplus C^{\infty}(M, T \mathcal{F}) .
$$

The compactly supported jets are denoted by $\mathfrak{J}_{0}^{\infty}$. For a jet $\mathfrak{u}=(a, u) \in \mathfrak{J}^{\infty}$ with scalar component $a$ and vector component $u$, we define $\nabla_{\mathfrak{u}}$ as the linear combination of scalar multiplication and directional derivative (1.8). Then the EL equations (2.5) clearly imply the so-called weak EL equations (1.9). The main difference to the strong EL equations is that in the weak formulation we restrict attention to the behavior of $\ell$ in an infinitesimal neighborhood of space-time $M$ (but in contrast to weak solutions of PDEs, "weak" does not refer to multiplying by a test function and integrating). Working with jets has the advantage that the two equations $\left.\ell\right|_{M}=0$ and $\left.D \ell\right|_{M}=0$ are combined in a single equation.

Adapting the notation (1.8) to second derivatives, we set

$$
\left.\nabla^{2} \ell\right|_{x}(\mathfrak{u}, \mathfrak{u}):=a(x)^{2} \ell(x)+2 a(x) D_{u} \ell(x)+D^{2} \ell \mid x(u, u) .
$$

In view of the weak EL equations (1.9), the zero and first order derivatives in this equation vanish for all $x \in M$. Therefore, inequality (3.1) implies that

$$
\left.\nabla^{2} \ell\right|_{x}(\mathfrak{u}, \mathfrak{u}) \geq 0 \quad \text { for all } x \in M .
$$

Integrating over $M$ gives the following result:

Proposition 3.1. Let $\rho$ be a minimizer of the causal action. Then

$$
\left.\int_{M} \nabla^{2} \ell\right|_{x}(\mathfrak{u}, \mathfrak{u}) d \rho(x) \geq 0 \quad \text { for all } \mathfrak{u} \in \mathfrak{J}_{0}^{\infty}
$$




\section{Positivity of Second Variations Generated by Jets}

In this section we analyze second variations for a a special class of variations of the measure $\rho$ to obtain another positive functional on jets. Similar as in [9, Section 3] we consider measures of the form

$$
\tilde{\rho}_{\tau}=\left(F_{\tau}\right)_{*}\left(f_{\tau} \rho\right) \quad \text { for } \tau \in\left(-\tau_{\max }, \tau_{\max }\right)
$$

with smooth mappings

$$
f \in C^{\infty}\left(\left(-\tau_{\max }, \tau_{\max }\right) \times M, \mathbb{R}^{+}\right) \quad \text { and } \quad F \in C^{\infty}\left(\left(-\tau_{\max }, \tau_{\max }\right) \times M, \mathcal{F}\right),
$$

where the star denotes the push-forward measure defined by $\left(\left(F_{\tau}\right)_{*} \mu\right)(\Omega)=\mu\left(F_{\tau}^{-1}(\Omega)\right)$ (where $\Omega \subset \mathcal{F}$; for basics see for example [2, Section 3.6]). We assume that for $\tau=0$ the variation is trivial,

$$
f_{0} \equiv 1 \quad \text { and } \quad F_{0} \equiv \mathbb{1} .
$$

Moreover, we assume that $F_{\tau}$ and $f_{\tau}$ are compactly supported, meaning that they are trivial outside a compact set $K \subset M$, i.e.

$$
\left.f_{\tau}\right|_{M \backslash K} \equiv 1 \quad \text { and }\left.\quad F_{\tau}\right|_{M \backslash K} \equiv \mathbb{1} .
$$

Finally, in order to satisfy the volume constraint on the right side of (2.2), we assume that

$$
\int_{K} f_{\tau}(x) d \rho(x)=\rho(K) \quad \text { for all } \tau \in\left(-\tau_{\max }, \tau_{\max }\right) .
$$

Then the transformation (4.1) is described infinitesimally by a smooth and compactly supported jet,

$$
\mathfrak{u}=(a, u):=\left(\dot{f}_{0}, \dot{F}_{0}\right) \in \mathfrak{J}_{0}^{\infty} .
$$

Moreover, differentiating the volume constraint (4.4) gives

$$
\int_{K} a(x) d \rho(x)=0 .
$$

We now compute the first and second variation of the action. Combining (2.3) with the definition of the push-forward measure, we obtain

$$
\begin{aligned}
\mathcal{S}\left(\tilde{\rho}_{\tau}\right)-\mathcal{S}(\rho)=2 & \int_{K} d \rho(x) \int_{M \backslash K} d \rho(y)\left(f_{\tau}(x) \mathcal{L}\left(F_{\tau}(x), y\right)-\mathcal{L}(x, y)\right) \\
& +\int_{K} d \rho(x) \int_{K} d \rho(y)\left(f_{\tau}(x) f_{\tau}(y) \mathcal{L}\left(F_{\tau}(x), F_{\tau}(y)\right)-\mathcal{L}(x, y)\right) .
\end{aligned}
$$

Then the first variation vanishes,

$$
\left.\frac{d}{d \tau} \mathcal{S}\left(\tilde{\rho}_{\tau}\right)\right|_{\tau=0}=2 \int_{K} d \rho(x) \int_{M} d \rho(y) \nabla_{1, \mathfrak{u}} \mathcal{L}(x, y)=2 \int_{K} \nabla_{\mathfrak{u}}\left(\ell(x)+\frac{\nu}{2}\right) d \rho(x)=0,
$$

where in the last step we used (1.9) and (4.5) (and $\nabla_{1}$ denotes the partial derivative acting on the first argument of the Lagrangian). Moreover, the second variation is computed by

$$
\begin{aligned}
& \left.\frac{d^{2}}{d \tau^{2}} \mathcal{S}\left(\tilde{\rho}_{\tau}\right)\right|_{\tau=0}=2 \int_{K} d \rho(x) \int_{K} d \rho(y) \nabla_{1, \mathfrak{u}} \nabla_{2, \mathfrak{u}} \mathcal{L}(x, y) \\
& +2 \int_{K} d \rho(x) \int_{M} d \rho(y)\left(a(x) D_{1, u} \mathcal{L}(x, y)+D_{1, u} D_{1, u} \mathcal{L}(x, y)+\left(\ddot{f}_{0}(x)+D_{1, \ddot{F}_{0}}\right) \mathcal{L}(x, y)\right) .
\end{aligned}
$$


In the last line we can carry out the $y$-integration using (2.4). Again combining the EL equations (1.9) with (4.5) (and a similar formula for $\ddot{f}$ ), we obtain the simple formula

$$
\left.\frac{1}{2} \frac{d^{2}}{d \tau^{2}} \mathcal{S}\left(\tilde{\rho}_{\tau}\right)\right|_{\tau=0}=\int_{K} d \rho(x) \int_{K} d \rho(y) \nabla_{1, \mathfrak{u}} \nabla_{2, \mathfrak{u}} \mathcal{L}(x, y)+\left.\int_{K} \nabla^{2} \ell\right|_{x}(\mathfrak{u}, \mathfrak{u}) d \rho(x) .
$$

Since $\rho$ is a minimizer and the first variation vanishes, the second variation is necessarily non-negative, giving rise to the inequality

$$
\int_{M} d \rho(x) \int_{M} d \rho(y) \nabla_{1, \mathfrak{u}} \nabla_{2, \mathfrak{u}} \mathcal{L}(x, y)+\left.\int_{M} \nabla^{2} \ell\right|_{x}(\mathfrak{u}, \mathfrak{u}) d \rho(x) \geq 0
$$

subject to the condition that the jet $\mathfrak{u}$ must satisfy the volume constraint (4.5). In the next proposition we remove this condition with a limiting procedure:

Proposition 4.1. Let $\rho$ be a minimizer of the causal action. Then

$$
\int_{M} d \rho(x) \int_{M} d \rho(y) \nabla_{1, \mathfrak{u}} \nabla_{2, \mathfrak{u}} \mathcal{L}(x, y)+\left.\int_{M} \nabla^{2} \ell\right|_{x}(\mathfrak{u}, \mathfrak{u}) d \rho(x) \geq 0 \quad \text { for all } \mathfrak{u} \in \mathfrak{J}_{0}^{\infty} .
$$

Proof. Let $\mathfrak{u}=(a, u) \in \mathfrak{J}_{0}^{\infty}$ be a jet which violates the volume constraint (4.5). Then, choosing a compact set $\Omega \subset M$ with $\rho(\Omega)>0$, the jet $\hat{\mathfrak{u}}:=(\hat{a}, u)$ with

$$
\hat{a}(x)=a(x)-c(\Omega) \chi_{\Omega}(x) \quad \text { and } \quad c(\Omega):=\frac{1}{\rho(\Omega)} \int_{\Omega} a(x) d \rho(x)
$$

(where $\chi_{\Omega}$ is the characteristic function) satisfies the volume constraint. Choosing the scalar variation $f_{\tau}=(1-\tau)+\tau \hat{a}$ and a family of diffeomorphisms $F_{\tau}$ with $\dot{F}_{0}=u$, we obtain a variation which satisfies the volume constraint (note that $\ddot{f}=0$ ). Clearly, due to the characteristic function, the jet $\hat{\mathfrak{u}}$ is no longer smooth, but it has again compact support, and an approximation argument using Lebesgue's dominated convergence theorem shows that the inequality (4.7) also holds for $\hat{\mathfrak{u}}$. Expanding in powers of $c$, we thus obtain the inequality

$$
\begin{array}{rl}
0 \leq \int_{K} & d \rho(x) \int_{K} d \rho(y) \nabla_{1, \mathfrak{u}} \nabla_{2, \mathfrak{u}} \mathcal{L}(x, y)+\left.\int_{K} \nabla^{2} \ell\right|_{x}(\mathfrak{u}, \mathfrak{u}) d \rho(x) \\
& -2 c \int_{M} d \rho(x) \int_{K} d \rho(y) \chi_{\Omega}(x) \nabla_{2, \mathfrak{u}} \mathcal{L}(x, y) \\
& +c^{2} \int_{M} d \rho(x) \int_{M} d \rho(y) \chi_{\Omega}(x) \chi_{\Omega}(y) \mathcal{L}(x, y) \\
& +\int_{M}\left(-2 c \chi_{\Omega}(x) \nabla_{\mathfrak{u}} \ell(x)+c^{2} \chi_{\Omega}(x)^{2} \ell(x)\right) d \rho(x)
\end{array}
$$

(the integrand in the last line arises from the contributions to $\nabla^{2} \ell \mid x(\mathfrak{u}, \mathfrak{u})$ involving the scalar components of the jets). The last line vanishes due to the weak EL equations (1.9). Hence

$$
\begin{aligned}
& \int_{K} d \rho(x) \int_{K} d \rho(y) \nabla_{1, \mathfrak{u}} \nabla_{2, \mathfrak{u}} \mathcal{L}(x, y)+\left.\int_{K} \nabla^{2} \ell\right|_{x}(\mathfrak{u}, \mathfrak{u}) d \rho(x) \\
& \geq 2 c \int_{K} d \rho(x) \int_{\Omega} d \rho(y) \nabla_{1, \mathfrak{u}} \mathcal{L}(x, y)-c^{2} \int_{K} d \rho(x) \int_{K} d \rho(y) \mathcal{L}(x, y)=: A(\Omega) .
\end{aligned}
$$

We now let $\left(\Omega_{n}\right)_{n \in \mathbb{N}}$ be an exhaustion of $M$ by compact sets. We distinguish the two cases when $\rho(M)$ is finite and infinite and treat these cases separately. If the 
total volume $\rho(M)$ is finite, one can take the limit $n \rightarrow \infty$ with Lebesgue's dominated convergence theorem to obtain

$$
\begin{gathered}
\lim _{n \rightarrow \infty} \int_{K} d \rho(x) \int_{\Omega_{n}} d \rho(y) \nabla_{1, \mathfrak{u}} \mathcal{L}(x, y)=\int_{K} d \rho(x) \int_{M} d \rho(y) \nabla_{1, \mathfrak{u}} \mathcal{L}(x, y) \\
\stackrel{(2.1)}{=} \int_{K} \nabla_{\mathfrak{u}}\left(\ell(x)+\frac{\nu}{2}\right) d \rho(x)=\frac{\nu}{2} \int_{K} a(x) d \rho(x) \\
\lim _{n \rightarrow \infty} A\left(\Omega_{n}\right)=2 c(M) \frac{\nu}{2} \int_{K} a(x) d \rho(x)-c(M)^{2} \rho(M) \frac{\nu}{2} \\
=\frac{\nu}{2 \rho(M)}\left(\int_{K} a(x) d \rho(x)\right)^{2} \geq 0
\end{gathered}
$$

where in the last line we substituted the value of $c(M)$ in (4.8).

In the remaining case that the volume $\rho(M)$ is infinite, we estimate the terms as follows,

$$
\begin{aligned}
& c\left(\Omega_{n}\right)^{2} \int_{K} d \rho(x) \int_{K} d \rho(y) \mathcal{L}(x, y) \\
& \leq c\left(\Omega_{n}\right)^{2} \int_{K} d \rho(x) \int_{M} d \rho(y) \mathcal{L}(x, y)=c\left(\Omega_{n}\right)^{2} \frac{\nu}{2} \rho(K) \rightarrow 0 \\
& \int_{K} d \rho(x) \int_{\Omega_{n}} d \rho(y) \nabla_{1, \mathfrak{u}} \mathcal{L}(x, y) \rightarrow \int_{K} d \rho(x) \int_{M} d \rho(y) \nabla_{1, \mathfrak{u}} \mathcal{L}(x, y) \\
& \stackrel{\text { (2.1) }}{=} \int_{K} \nabla_{\mathfrak{u}}\left(\ell(x)+\frac{\nu}{2}\right) d \rho(x)=\frac{\nu}{2} \int_{K} a(x) d \rho(x) .
\end{aligned}
$$

As a consequence, $A\left(\Omega_{n}\right)$ converges to zero as $n \rightarrow \infty$. This concludes the proof.

We close this section with two remarks.

Remark 4.2. (scalar jets) In the special case $\mathfrak{u}=(a, 0)$ of a purely scalar jet, the statement of Proposition 4.1 simplifies to the inequality

$$
\int_{M} d \rho(x) \int_{M} d \rho(y) a(x) a(y) \mathcal{L}(x, y) \geq 0 \quad \text { for all } a \in C_{0}^{\infty}(M, \mathbb{R}) .
$$

In the compact setting, this inequality was already derived in [10, Lemma 3.5]. It is a main ingredient in the analysis of the singular support in [10, Section 3] (see [10, Theorems 3.16 and 3.18]). Furthermore, in [1, Theorem 3.16] a similar positivity statement was derived in the setting of causal fermion systems.

Moreover, second variations are studied in [9, Section 4.4], and a stability result is obtained (see [9, Proposition 4.10]). In this analysis, only variations $\left(\tilde{\rho}_{\tau}\right)_{\tau \in\left(-\tau_{\max }, \tau_{\max }\right)}$ are considered where $\mu_{\tau}:=\tilde{\rho}_{\tau}-\rho$ is a smooth curve in the Banach space of signed measures of bounded total variation. In particular, the derivatives $\dot{\tilde{\rho}}_{0}$ and $\ddot{\tilde{\rho}}_{0}$ are assumed to be signed measures. Such variations do not include variations of the form (4.1) (because for example the infinitesimal transport of a Dirac measure gives a distributional derivative of a Dirac measure, which is not a signed measure). This is the reason why, using the language of jet spaces, only the scalar component of the jets comes into play. As a consequence, the stability result follows already by analyzing the inequality for scalar jets (4.9). 
To summarize, the new feature in Proposition 4.1 is the inclusion of the vector component of the jets. This is a major improvement which gives much more information, in particular if the dimension of $\mathcal{F}$ is large.

Remark 4.3. (adding points to the support) We now discuss the situation that there is a point $y \in \mathcal{F} \backslash M$ with $\ell(y)=0$. In the setting of causal fermion systems, this case was treated in [1, Section 3.5] working with moment measures. For self-consistency, we here repeat the argument in our setting and explain why the jet formalism does not make it possible to improve the result.

More generally, let $A \subset \mathcal{F}$ be a compact set with

$$
A \cap M=\varnothing \quad \text { and }\left.\quad \ell\right|_{A} \equiv 0
$$

(a typical choice is $A=\{y\}$ ). We choose a measure $\mu$ on $A$ with $\mu(A)<\infty$. Moreover, we choose a function $a \in C_{0}^{\infty}\left(M \cup A, \mathbb{R}_{0}^{+}\right)$with

$$
\left.a\right|_{A} \geq 0 \quad \text { and } \quad \int_{F} a(x) d(\rho+\mu)(x)=0 .
$$

We now consider the variation

$$
\tilde{\rho}_{\tau}=(1-\tau a) \rho+\tau a \mu \quad \tau \in[0,1) .
$$

Then the first variation vanishes because $\ell$ vanishes identically on the support of $a$. Hence the second variation must be non-negative. Evaluating this condition gives the result of [1, Theorem 3.17].

In order to analyze the effect of a vector components of the jets, we consider the more general variation

$$
\tilde{\rho}_{\tau}=\left(F_{\tau}\right)_{*}((1-\tau a) \rho+\tau a \mu)
$$

with a mapping $F_{\tau} \in C^{\infty}(M \cup A, \mathcal{F})$ which we again assume to be trivial outside a compact set. Infinitesimally, this variation is described by the jet

$$
\mathfrak{u}=\left(a, \dot{F}_{0}\right) \in C_{0}^{\infty}\left(M \cup A, \mathbb{R}_{0}^{+}\right) \oplus C_{0}^{\infty}(M \cup A, T \mathcal{F})
$$

The first variation again vanishes. Thus the second variation must again be nonnegative. On the set $A$, the second variation necessarily involves the scalar component (because without the scalar component, $\tilde{\rho}_{\tau}$ vanishes on $A$ ). As a consequence, the second variation involves the vector component on $A$ at most linearly. Therefore, using a block matrix notation where the first component contains the jets in $M$ as well as the scalar component in $A$ and the second component contains the vector component in $A$, the second variations are described by a bilinear form with the structure

$$
\left\langle\mathfrak{u},\left(\begin{array}{cc}
L_{11} & L_{12} \\
L_{21} & 0
\end{array}\right) \mathfrak{u}\right\rangle
$$

with suitable operators $L_{i j}$. The positivity of this bilinear form is equivalent to the positivity of the upper left matrix entry. This shows that second variations of the form (4.10) do not give more information than [1, Theorem 3.17] and Proposition 4.1 above. 


\section{Stability under Variations with Local Fragmentation}

We now consider variations of the general form (1.7), where the weight functions $c_{\mathfrak{a}}$ are normalized at every point, i.e.

$$
c_{\mathfrak{a}}(x) \geq 0 \quad \text { and } \quad \sum_{\mathfrak{a}=1}^{L} c_{\mathfrak{a}}(x)=1 \quad \text { for all } x \in M .
$$

In order to keep the setting as simple as possible, we assume that the functions $c_{\mathfrak{a}}$ are smooth. Moreover, similar to (4.2) and (4.3), we assume that that for every $\mathfrak{a}$, the transformation is trivial for $\tau=0$ and is trivial outside a compact set $K$. Then our variation is described infinitesimally by jets $\mathfrak{u}_{\mathfrak{a}}=\left(b_{\mathfrak{a}}, u_{\mathfrak{a}}\right) \in \mathfrak{J}_{0}^{\infty}$. In analogy to (4.5), the volume constraint becomes

$$
\int_{K} \sum_{\mathfrak{a}=1}^{L} c_{\mathfrak{a}}(x) b_{\mathfrak{a}}(x) d \rho(x)=0 .
$$

We remark that we here consider local fragmentations, meaning that we allow the weights $c_{\mathfrak{a}}$ to be functions of $x$. This is different from the procedure in [6, Section 5], where the weights $c_{\mathfrak{a}}$ were real parameters. However, this is not an important difference, because after approximating the weight functions $c_{\mathfrak{a}}(x)$ by locally constant functions taking values in $\mathbb{N} / \tilde{L}$ with large $\tilde{L}$ and by increasing the number of subsystems to $\tilde{L}$, one can approximate a variation involving local fragmentation by variations with constant weights $c_{\mathfrak{a}}=1 / \tilde{L}$. Therefore, considering local fragmentation is mainly a matter of convenience and has the advantage of giving a somewhat different perspective.

The variation of the action can be computed similar as in Section 4. The first variation again vanishes (as is obvious by linearity in the jets). When computing the second variation, the terms involving up to first derivatives of $\mathcal{L}$ again drop out. We thus obtain in generalization of (4.6)

$$
\begin{aligned}
\left.\frac{1}{2} \frac{d^{2}}{d \tau^{2}} \mathcal{S}\left(\tilde{\rho}_{\tau}\right)\right|_{\tau=0}= & \int_{K} d \rho(x) \int_{K} d \rho(y) \sum_{\mathfrak{a}, \mathfrak{b}=1}^{L} c_{\mathfrak{a}}(x) c_{\mathfrak{b}}(y) \nabla_{1, \mathfrak{u}_{\mathfrak{a}}} \nabla_{2, \mathfrak{u}_{\mathfrak{b}}} \mathcal{L}(x, y) \\
& +\left.\int_{K} \sum_{\mathfrak{a}=1}^{L} c_{\mathfrak{a}}(x) \nabla^{2} \ell\right|_{x}\left(\mathfrak{u}_{\mathfrak{a}}, \mathfrak{u}_{\mathfrak{a}}\right) d \rho(x) .
\end{aligned}
$$

In order to clarify the scaling behavior in the weights $c_{\mathfrak{a}}$, it is useful to transform the jets according to $\mathfrak{u}_{\mathfrak{a}} \rightarrow \mathfrak{u}_{\mathfrak{a}} / c_{\mathfrak{a}}$ (thus we multiply both the scalar and vector components of the jet $\mathfrak{u}$ by the same function $c_{\mathfrak{a}}(x)$; this is admissible because this function is smooth and bounded, so that multiplying by it again gives a jet in $\left.\mathfrak{J}_{0}^{\infty}:=C_{0}^{\infty}(M, \mathbb{R}) \oplus C_{0}^{\infty}(M, T \mathcal{F})\right)$. We thus obtain the inequality

$$
\begin{array}{rl}
0 \leq\left.\frac{1}{2} \frac{d^{2}}{d \tau^{2}} \mathcal{S}\left(\tilde{\rho}_{\tau}\right)\right|_{\tau=0}=\int_{K} & d \rho(x) \int_{K} d \rho(y) \sum_{\mathfrak{a}, \mathfrak{b}=1}^{L} \nabla_{1, \mathfrak{u}_{\mathfrak{a}}} \nabla_{2, \mathfrak{u}_{\mathfrak{b}}} \mathcal{L}(x, y) \\
& +\left.\int_{K} \sum_{\mathfrak{a}=1}^{L} \frac{1}{c_{\mathfrak{a}}(x)} \nabla^{2} \ell\right|_{x}\left(\mathfrak{u}_{\mathfrak{a}}, \mathfrak{u}_{\mathfrak{a}}\right) d \rho(x)
\end{array}
$$

where the weights appear only in the last summand. Now it is apparent that the positivity of the second variation yields the positivity statements of both Section 3 and 
Section 4. Indeed, in the case $L=1$ of one subsystem we clearly get back to the setting of Section 4. Moreover, in the limiting case $c_{1} \searrow 0$ and $c_{2}=\ldots=c_{L} \approx(L-1)^{-1}$, the factor $c_{1}^{-1}$ in (5.2) diverges, implying that

$$
\left.\int_{K} \nabla^{2} \ell\right|_{x}\left(\mathfrak{u}_{1}, \mathfrak{u}_{1}\right) d \rho(x) \geq 0 .
$$

We thus recover the result of Proposition 3.1.

In order to understand what the inequality (5.2) means, it is helpful to choose the weights $c_{\mathfrak{a}}$ at every point in such a way that the last summand in (5.2) becomes as small as possible, because then the inequality in (5.2) gives most information. Abbreviating the integrand by $A_{\mathfrak{a}}=\left.\nabla^{2} \ell\right|_{x}\left(\mathfrak{u}_{\mathfrak{a}}, \mathfrak{u}_{\mathfrak{a}}\right) \geq 0$, our task is to

$$
\operatorname{minimize} \sum_{\mathfrak{a}=1}^{L} \frac{A_{\mathfrak{a}}}{c_{\mathfrak{a}}}
$$

under the constraints (5.1). The method of Lagrange multipliers gives

$$
c_{\mathfrak{a}}=\sqrt{\frac{A_{\mathfrak{a}}}{\lambda}} \quad \text { with } \quad \lambda=\left(\sum_{\mathfrak{a}} \sqrt{A_{\mathfrak{a}}}\right)^{2} .
$$

A direct computation shows that this choice of weights satisfies (5.1). Moreover, the following consideration shows that this choice of weights indeed realizes the minimum: Clearly, if one of the parameters $A_{\mathfrak{a}}$ vanishes, then the minimum is attained in the limiting case $c_{\mathfrak{a}} \searrow 0$. With this in mind, we may assume that all the $A_{\mathfrak{a}}$ are strictly positive. Next, at the boundary of the admissible range for the parameters $\left\{c_{1}, \ldots, c_{L}\right\}$ one or several of the coefficients $c_{\mathfrak{a}}$ vanish. As a consequence, the expression in (5.3) tends to $+\infty$ at the boundary of the admissible parameter range. It follows that the only interior critical point is the absolute minimum.

Substituting the obtained values for $c_{\mathfrak{a}}$ into (5.2), we obtain the inequality

$$
\begin{gathered}
0 \leq \int_{K} d \rho(x) \int_{K} d \rho(y) \sum_{\mathfrak{a}, \mathfrak{b}=1}^{L} \nabla_{1, \mathfrak{u}_{\mathfrak{a}}} \nabla_{2, \mathfrak{u}_{\mathfrak{b}}} \mathcal{L}(x, y) \\
+\int_{K}\left(\sum_{\mathfrak{a}=1}^{L} \sqrt{\left.\nabla^{2} \ell\right|_{x}\left(\mathfrak{u}_{\mathfrak{a}}, \mathfrak{u}_{\mathfrak{a}}\right)}\right)^{2} d \rho(x) .
\end{gathered}
$$

The inequality (5.2) holds for all choices of the weight functions if and only if (5.4) holds.

From the inequality (5.4) one can read off our main stability result:

Proof of Theorem 1.1. The inequalities (1.10) and (1.11) were derived in Propositions 3.1 and 4.1. In order to derive the stability statement, let $\rho$ be a Radon measure which satisfies (1.9) as well as the inequalities (1.10) and (1.11). Moreover, assume that the inequality (1.11) is strict for every non-zero $\mathfrak{u} \in \mathfrak{J}_{0}^{\infty}$.

Let $\left(\tilde{\rho}_{\tau}\right)_{\tau \in\left(-\tau_{\max }, \tau_{\max }\right)}$ be a non-trivial variation with local fragmentation (1.7). The inequality (1.10) allows us to introduce a seminorm on the jets by

$$
\|\mathfrak{u}(x)\|:=\sqrt{\left.\nabla^{2} \ell\right|_{x}(\mathfrak{u}, \mathfrak{u})} .
$$


Using the strict inequality in (1.11), we obtain

$$
\begin{aligned}
& -\int_{K} d \rho(x) \int_{K} d \rho(y) \sum_{\mathfrak{a}, \mathfrak{b}=1}^{L} \nabla_{1, \mathfrak{u}_{\mathfrak{a}}} \nabla_{2, \mathfrak{u}_{\mathfrak{b}}} \mathcal{L}(x, y) \\
& \quad<\left.\int_{K} \nabla^{2} \ell\right|_{x}\left(\sum_{\mathfrak{a}=1}^{L} \mathfrak{u}_{\mathfrak{a}}, \sum_{\mathfrak{b}=1}^{L} \mathfrak{u}_{\mathfrak{b}}\right)=\int_{K}\left\|\sum_{\mathfrak{a}=1}^{L} \mathfrak{u}_{\mathfrak{a}}\right\|^{2} \\
& \quad \stackrel{(*)}{\leq} \int_{K}\left(\sum_{\mathfrak{a}=1}^{L}\left\|\mathfrak{u}_{\mathfrak{a}}(x)\right\|\right)^{2}=\int_{K}\left(\sum_{\mathfrak{a}=1}^{L} \sqrt{\left.\nabla^{2}(x) \ell\right|_{x}\left(\mathfrak{u}_{\mathfrak{a}}, \mathfrak{u}_{\mathfrak{a}}\right)}\right)^{2},
\end{aligned}
$$

where in $(*)$ we used the triangle inequality for the seminorm (5.5). As a consequence, the inequality (5.4) is strict, which implies that also the inequality (5.2) is strict. This concludes the proof.

\section{Application: Hilbert Spaces of Jets}

As an application, we now explain how our positive functionals can be used to endow the space of jets in space-time with Hilbert space structures. These Hilbert space structures should be very useful because they make functional analytic tools applicable to the analysis of the jet spaces and the causal action principle. We now introduce the following bilinear forms on $\mathfrak{J}_{0}^{\infty}$ :

$$
\begin{aligned}
\langle\mathfrak{u}, \mathfrak{v}\rangle & :=\int_{M} d \rho(x) \int_{M} d \rho(y) \nabla_{1, \mathfrak{u}} \nabla_{2, \mathfrak{v}} \mathcal{L}(x, y)+\left.\int_{M} \nabla^{2} \ell\right|_{x}(\mathfrak{u}, \mathfrak{v}) d \rho(x) \\
\langle\langle\mathfrak{u}, \mathfrak{v}\rangle\rangle & :=\langle\mathfrak{u}, \mathfrak{v}\rangle+\left.\int_{M} \nabla^{2} \ell\right|_{x}(\mathfrak{u}, \mathfrak{v}) d \rho(x) .
\end{aligned}
$$

By Theorem 1.1, both bilinear forms are positive semi-definite. Thus dividing out the null space and forming the completion gives real Hilbert spaces of jets denoted by $\mathcal{H}\langle\ldots .$, and $\mathcal{H}\langle\langle\cdot, \cdot\rangle$, respectively. Obviously,

$$
\langle\mathfrak{u}, \mathfrak{u}\rangle \leq\langle\langle\mathfrak{u}, \mathfrak{u}\rangle\rangle,
$$

giving rise to a norm-decreasing mapping $\mathcal{H}\langle\langle.,\rangle.\rangle \rightarrow \mathcal{H}^{\langle\cdot, \cdot,\rangle}$.

For the scalar components of the jets, the two scalar products (6.1) and (6.2) obviously agree. But they are quite different for the vector components. In order to understand this difference, it is instructive to consider a jet $\mathfrak{u}=(0, u)$ which describes a symmetry of the Lagrangian, i.e. (for details see [8, Section 3.1])

$$
\left(D_{1, u}+D_{2, u}\right) \mathcal{L}(x, y)=0 \quad \text { for all } x, y \in M .
$$

For this jet, a direct computation shows that

$$
\langle\mathfrak{u}, \mathfrak{u}\rangle=0 .
$$

Hence symmetry transformations lie in the kernel of the bilinear form $\langle.,$.$\rangle and thus$ correspond to the zero vector in $\mathcal{H}^{\langle. . .\rangle}$. For example, in the setting of causal fermion systems, jets describing global phase transformations (see [8, Section 5.1]) are not contained in $\mathcal{H}^{\langle\ldots . .\rangle}$. Generally speaking, the scalar product $\langle.,$.$\rangle makes it possible$ to disregard symmetry transformations of the causal fermion system. However, jets describing symmetry transformations do in general correspond to non-zero vectors of the Hilbert space $\mathcal{H}\langle(.,$.$\rangle .$ 


\section{Application: A Positive Surface Layer Integral}

In the setting of causal variational principles, the usual integrals over hypersurfaces in space-time are undefined. Instead, one considers so-called surface layer integrals. In general terms, a surface layer integral is a double integral of the form

$$
\int_{\Omega} d \rho(x) \int_{M \backslash \Omega} d \rho(y) \cdots \mathcal{L}(x, y)
$$

where $\Omega$ is a subset of $M$ and $\cdots$ stands for a differential operator acting on the Lagrangian. The structure of such surface layer integrals can be understood most easily in the special situation that the Lagrangian is of short range in the sense that $\mathcal{L}(x, y)$ vanishes unless $x$ and $y$ are close together. In this situation, we only get a contribution to the double integral (7.1) if both $x$ and $y$ are close to the boundary $\partial \Omega$. With this in mind, surface layer integrals can be understood as an adaptation of surface integrals to the setting of causal variational principles (for a more detailed explanation see 8 , Section 2.3]).

In [8], it is shown that there are conservation laws expressed in terms of surface layer integrals which generalize the well-known charge and current conservation. In [9], a conserved surface layer integral was found which generalizes the symplectic form to the setting of causal variational principles. We now derive a surface layer integral which is not necessarily conserved, but which has a definite sign. A jet $\mathfrak{v} \in \mathfrak{J}$ (not necessarily with compact support) is referred to as a solution of the linearized field equations if it satisfies the equation (for details and the motivation see [9])

$$
\nabla_{\mathfrak{u}}\left(\int_{M}\left(\nabla_{1, \mathfrak{v}}+\nabla_{2, \mathfrak{v}}\right) \mathcal{L}(x, y) d \rho(y)-\nabla_{\mathfrak{v}} \frac{\nu}{2}\right)=0
$$

for all $\mathfrak{u} \in \mathfrak{J}^{\infty}$ and all $x \in M$.

The following proposition shows that there is a positive surface layer integral. Similar as explained at the beginning of Section 6 , this can be used to endow the jet space with a Hilbert structure. But in contrast to the scalar products in Section 6 , where the jets were integrated over space-time, here the scalar product is given as a surface layer integral. This should be very useful for analyzing the dynamics of jets in space-time.

Proposition 7.1. Assume that $\mathfrak{v}$ is a solution of the linearized field equations (7.2). Then for any compact $\Omega \subset M$, the following surface layer integral is positive,

$$
-\int_{\Omega} d \rho(x) \int_{M \backslash \Omega} d \rho(y) \nabla_{1, \mathfrak{v}} \nabla_{2, \mathfrak{v}} \mathcal{L}(x, y) \geq 0 .
$$

Proof. Denoting the components of $\mathfrak{v}$ by $\mathfrak{v}=(b, v)$, we evaluate (7.2) for $\mathfrak{u}=\mathfrak{v}$ and integrate over $\Omega$. The resulting integrals can be rewritten as follows,

$$
\begin{aligned}
0= & \int_{\Omega} d \rho(x) \int_{M} d \rho(y) \nabla_{1, \mathfrak{v}}\left(\nabla_{1, \mathfrak{v}}+\nabla_{2, \mathfrak{v}}\right) \mathcal{L}(x, y)-\frac{\nu}{2} \int_{\Omega} b(x)^{2} d \rho(x) \\
= & \left.\int_{\Omega} \nabla^{2} \ell\right|_{x}(\mathfrak{v}, \mathfrak{v}) d \rho(x)+\int_{\Omega} d \rho(x) \int_{M} d \rho(y) \nabla_{1, \mathfrak{v}} \nabla_{2, \mathfrak{v}} \mathcal{L}(x, y) \\
= & \left.\int_{\Omega} \nabla^{2} \ell\right|_{x}(\mathfrak{v}, \mathfrak{v}) d \rho(x)+\int_{\Omega} d \rho(x) \int_{\Omega} d \rho(y) \nabla_{1, \mathfrak{v}} \nabla_{2, \mathfrak{v}} \mathcal{L}(x, y) \\
& \quad+\int_{\Omega} d \rho(x) \int_{M \backslash \Omega} d \rho(y) \nabla_{1, \mathfrak{v}} \nabla_{2, \mathfrak{v}} \mathcal{L}(x, y) .
\end{aligned}
$$


Approximating the jet $\chi_{\Omega} \mathfrak{v}$ in (7.3) by smooth jets with compact support, one finds that the integrals in (7.3) are non-negative by Proposition 4.1. This gives the result.

We finally remark that in [4, Section 6] the surface layer integral in the last proposition is computed in Minkowski space.

Acknowledgments: I would like to thank Magdalena Lottner and the referees for helpful comments on the manuscript.

\section{REFERENCES}

[1] Y. Bernard and F. Finster, On the structure of minimizers of causal variational principles in the non-compact and equivariant settings, arXiv:1205.0403 [math-ph], Adv. Calc. Var. 7 (2014), no. 1, $27-57$.

[2] V.I. Bogachev, Measure Theory. Vol. I, Springer-Verlag, Berlin, 2007.

[3] F. Finster, The Continuum Limit of Causal Fermion Systems, arXiv:1605.04742 [math-ph], Fundamental Theories of Physics, vol. 186, Springer, 2016.

[4] - The causal action in Minkowski space and surface layer integrals, arXiv:1711.07058 [math-ph] (2017).

[5] _ Causal fermion systems: A primer for Lorentzian geometers, arXiv:1709.04781 [math-ph] (2017).

[6] _ Perturbation theory for critical points of causal variational principles, arXiv:1703.05059 [math-ph] (2017).

[7] F. Finster and J. Kleiner, Causal fermion systems as a candidate for a unified physical theory, arXiv:1502.03587 [math-ph], J. Phys.: Conf. Ser. 626 (2015), 012020.

[8] - Noether-like theorems for causal variational principles, arXiv:1506.09076 [math-ph], Calc. Var. Partial Differential Equations 55:35 (2016), no. 2, 41.

[9] _ A Hamiltonian formulation of causal variational principles, arXiv:1612.07192 [math-ph], Calc. Var. Partial Differential Equations 56:73 (2017), no. 3, 33.

[10] F. Finster and D. Schiefeneder, On the support of minimizers of causal variational principles, arXiv:1012.1589 [math-ph], Arch. Ration. Mech. Anal. 210 (2013), no. 2, 321-364.

[11] H.H. Goldstine, A History of the Calculus of Variations from the 17th Through the 19th Century, Studies in the History of Mathematics and Physical Sciences, vol. 5, Springer-Verlag, New YorkBerlin, 1980.

[12] P.R. Halmos, Measure Theory, Springer, New York, 1974.

[13] W. Rudin, Real and Complex Analysis, third ed., McGraw-Hill Book Co., New York, 1987.

Fakultät für Mathematik, Universität Regensburg, D-93040 Regensburg, Germany

E-mail address: finster@ur.de 